\title{
Accuracy of a Robotic Endoscopic System in Cadaver Models with Simulated Tumor Targets: ACCESS Study
}

\author{
Alexander C. Chen ${ }^{\mathrm{a}}$ Nicholas J. Pastis ${ }^{\mathrm{b}}$ Michael S. Machuzak ${ }^{\mathrm{c}}$ \\ Thomas R. Gildea ${ }^{c}$ Michael J. Simoff ${ }^{d}$ Colin T. Gillespie $^{\mathrm{e}}$ Amit K. Mahajan ${ }^{f}$ \\ Scott S. Oh' Gerard A. Silvestrib \\ aDivision of Pulmonary and Critical Care Medicine, Washington University School of Medicine, St. Louis, MO, USA; \\ ${ }^{b}$ Division of Pulmonary and Critical Care Medicine, Medical University of South Carolina, Charleston, SC, USA; \\ ${ }^{\mathrm{C}}$ Respiratory Institute, Cleveland Clinic, Cleveland, $\mathrm{OH}, \mathrm{USA} ;{ }^{\mathrm{d}}$ Division of Pulmonary and Critical Care Medicine, \\ Henry Ford Hospital, Wayne State University School of Medicine, Detroit, MI, USA; ${ }^{e}$ Division of Pulmonary and \\ Critical Care Medicine, Northwestern University Feinberg School of Medicine, Chicago, IL, USA; ${ }^{f}$ Inova Heart \\ and Vascular Institute, Falls Church, VA, USA; ${ }^{9}$ Division of Pulmonary Medicine, University of California, Los Angeles, \\ CA, USA
}

\section{Keywords}

Bronchoscopy · Pulmonary nodules · Robotic endoscopy

\begin{abstract}
Background: Bronchoscopy for the diagnosis of peripheral pulmonary lesions continues to present clinical challenges, despite increasing experience using newer guided techniques. Robotic bronchoscopic platforms have been developed to potentially improve diagnostic yields. Previous studies in cadaver models have demonstrated increased reach into the lung periphery using robotic systems compared to similarly sized conventional bronchoscopes, although the clinical impact of additional reach is unclear. $\mathbf{O b}$ jectives: This study was performed to evaluate the performance of a robotic bronchoscopic system's ability to reach and access artificial tumor targets simulating peripheral nodules in human cadaveric lungs. Methods: Artificial tumor targets sized $10-30 \mathrm{~mm}$ in axial diameter were implanted
\end{abstract}

into 8 human cadavers. CT scans were performed prior to procedures and all cadavers were intubated and mechanically ventilated. Electromagnetic navigation, radial probe endobronchial ultrasound, and fluoroscopy were used for all procedures. Robotic-assisted bronchoscopy was performed on each cadaver by an individual bronchoscopist to localize and biopsy peripheral lesions. Results: Sixty-seven nodules were evaluated in 8 cadavers. The mean nodule size was 20.4 $\mathrm{mm}$. The overall diagnostic yield was 65/67 (97\%) and there was no statistical difference in diagnostic yield for lesions $<20 \mathrm{~mm}$ compared with lesions measuring $21-30 \mathrm{~mm}$, the presence of a concentric or eccentric radial ultrasound image, or relative distance from the pleura. Conclusions: The robotic bronchoscopic system was successful at biopsying $97 \%$ of peripheral pulmonary lesions $10-30 \mathrm{~mm}$ in size in human cadavers. These findings support further exploration of this technology in prospective clinical trials in live human subjects.

(c) 2019 The Author(s)

Published by S. Karger AG, Basel

\begin{tabular}{ll}
\hline KARGER & $\begin{array}{l}\text { Ko } 2019 \text { The Author(s) } \\
\text { Published by S. Karger AG, Basel }\end{array}$ \\
Open ciccess \\
E-Mail karger@karger.com & This article is licensed under the Creative Commons Attribution- \\
wwww.karger.com/res & $\begin{array}{l}\text { NonCommercial-NoDerivatives 4.0 International License (CC BY- } \\
\text { NC-ND) (http://www.karger.com/Services/OpenAccessLicense). } \\
\text { Usage and distribution for commercial purposes as well as any dis- } \\
\text { tribution of modified material requires written permission. }\end{array}$
\end{tabular}

Alexander C. Chen, MD

Divisions of Pulmonary and Critical Care Medicine and Cardiothoracic Surgery Washington University School of Medicine

Campus Box 8052, 4523 Clayton Avenue, St. Louis, MO 63110-1093 (USA)

E-Mail chenac@wustl.edu 


\section{Introduction}

The successful diagnosis of peripheral pulmonary lesions continues to present clinical challenges, particularly for smaller pulmonary nodules. Despite technical advancements, including image guidance during bronchoscopy using electromagnetic navigation and ultrasoundbased approaches such as radial probe endobronchial ultrasound, no singular approach has demonstrated consistently high yields $[1,2]$.

One potential limitation of current approaches using conventional bronchoscopes may be reach into the lung periphery. As the bronchial tree narrows with each generation, the ability to advance conventional bronchoscopes into close proximity to targeted nodules may be suboptimal.

Another potential limitation of conventional bronchoscopy may be control and precision of instrumentation at the time of biopsy. Multiple publications have reported that the diagnostic yield of guided bronchoscopy is significantly higher when the peripheral lesion surrounds the bronchus compared to when the lesion is adjacent to the nearest bronchus [3-5]. Failure of current approaches may in part be due to the lack of fine motor control of instrumentation in the periphery.

Robotic bronchoscopic platforms have been developed as a method to potentially supplement the bronchoscopists' ability to reach and access peripheral lung lesions $[6,7]$. These systems have redesigned scope configurations that provide additional structural support as well as increased articulation points designed to potentially improve peripheral lesion biopsies.

We have previously demonstrated improved reach into the periphery of human cadaveric lungs using a robotic bronchoscopic platform when compared to a conventional thin bronchoscope of identical outer diameter, though the clinical implications of added reach on diagnostic yield are unclear [8]. We performed this study to further evaluate a robotic bronchoscopic system's ability to reach and access artificial tumor targets simulating peripheral nodules in human cadaveric lungs.

\section{Methods}

Robotic-assisted bronchoscopy procedures were performed on 8 cadavers by 8 bronchoscopists (A.C.C., N.J.P., M.S.M., T.R.G., M.J.S., C.T.G., A.K.M., S.S.O.) using the Monarch platform (Auris Health, Redwood City, CA, USA) between March 21 and April 18, 2019.

\section{Robotic Bronchoscopic System}

The robotic bronchoscopic system is composed of a tower with a monitor as well as a unit that houses automated arms that control insertion, retraction, and articulation of an outer sheath $(6.0 \mathrm{~mm}$ outer diameter) and inner scope (4.4 mm outer diameter) that has a 2.1-mm working channel. The sheath and scope are driven using a handheld controller and may be operated simultaneously as a unit, or independently (Fig. 1).

\section{Cadaver Preparation}

Eight fresh cadavers with intact head and torsos were prepared for this study. Artificial tumor targets were created using a mixture of agar, gelatin, iodinated contrast, colored mica powder, and wa-
Fig. 1. Robotic bronchoscopic system. a Arms and tower. b Sheath and scope. c Controller.

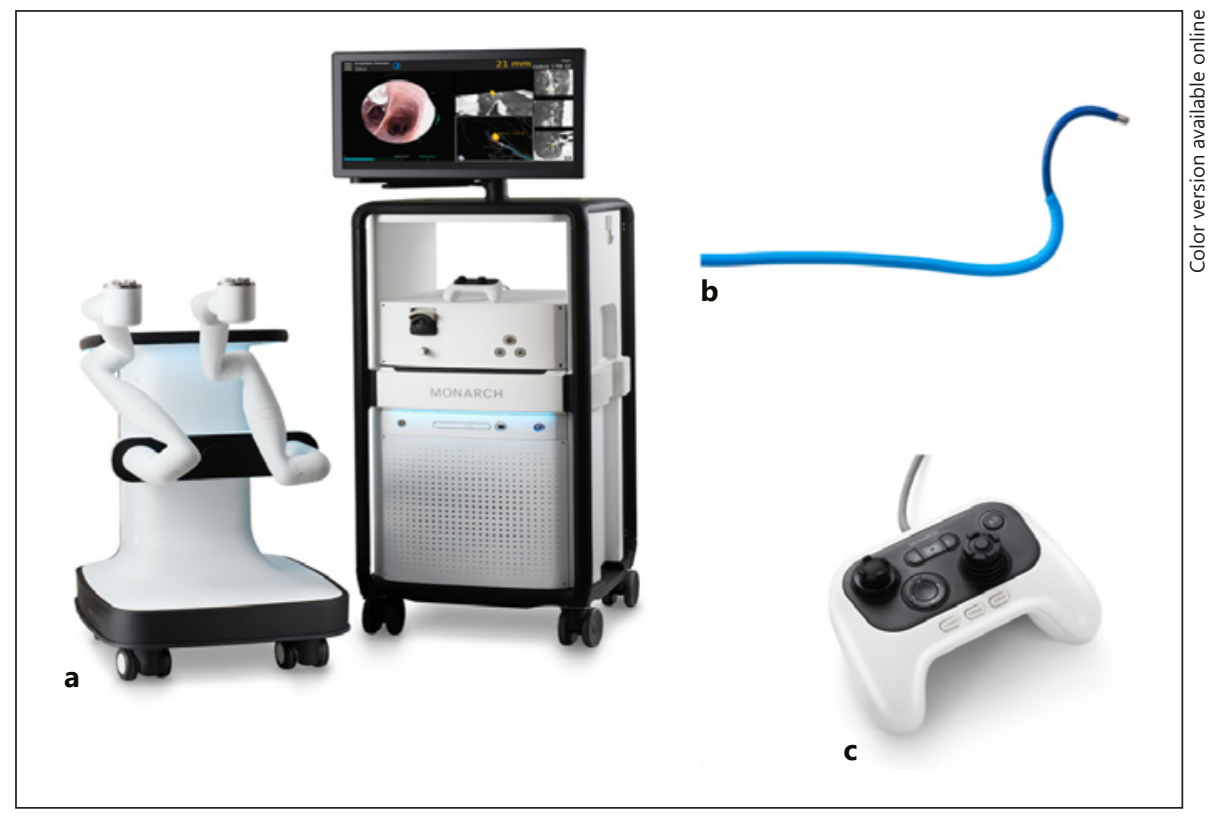

Robotic Bronchoscopy for Simulated Tumor Targets
Respiration 2020;99:56-61 DOI: $10.1159 / 000504181$ 
Fig. 2. CT images of implanted tumor targets (arrows) within cadavers.

3

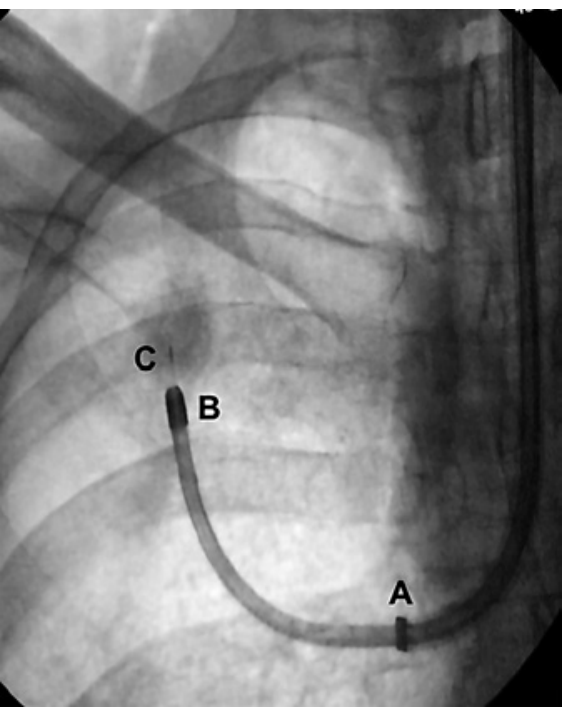

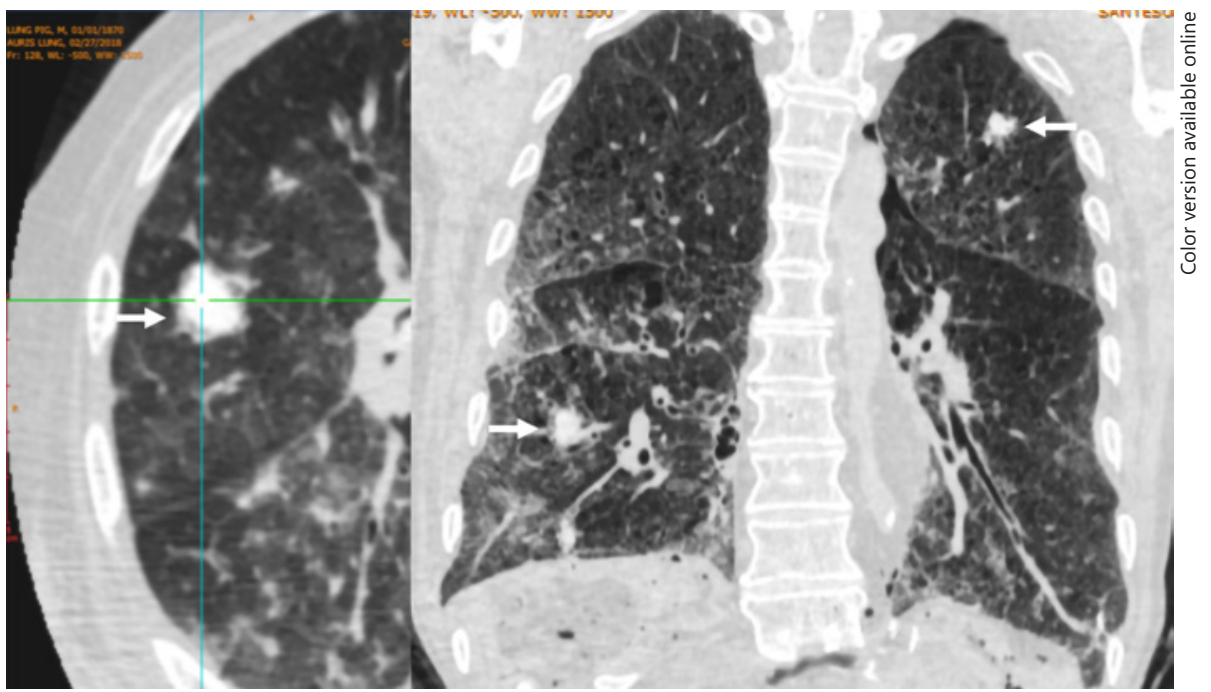

4

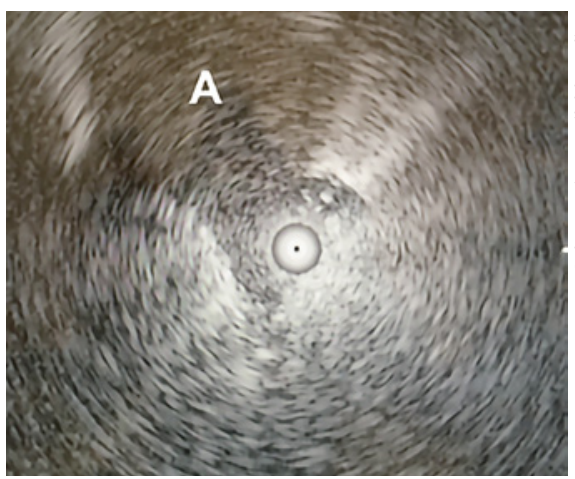

5

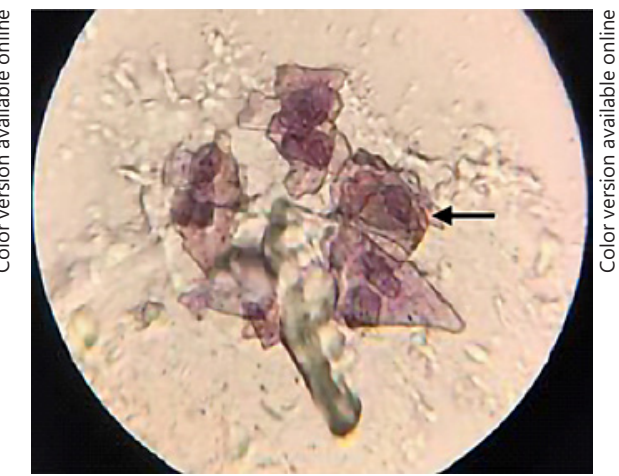

Fig. 3. Fluoroscopic image of the robotic system with TBNA of a RUL lesion: sheath tip (A), scope tip (B), and needle (C).

Fig. 4. Eccentric radial ultrasound image of an implanted lesion (A) within a cadaver.

Fig. 5. Microscopic image of a diagnostic TBNA specimen demonstrating pigmented powder (arrow). ter heated to an aqueous solution which was injected transthoracically into the lung parenchyma using fluoroscopic guidance. This technique was modified from a previously validated approach creating tumor "targets" that produce a realistic ultrasound image of tissue dense lesions that are visible on CT scanning [9]. The relative distance from the visceral pleura was estimated at the time of injection to provide a heterogenous distribution of peripheral pulmonary lesions approximately $0-30 \mathrm{~mm}$ from the pleura. The volume of material injected was controlled to create lesions $10-30 \mathrm{~mm}$ in diameter as measured on axial CT imaging. A minimum of 8 targets were injected into each cadaver. Thin-slice CT scans were subsequently performed on all cadavers (Fig. 2).

\section{Training}

Prior to study procedures, all 8 bronchoscopists performed robotic bronchoscopy on at least 4 cadavers without implanted tumor targets to gain familiarity with the system. During these pro- cedures, the bronchoscopists received training from Auris team members regarding manipulation of the system and performing biopsies. During study cases, no external assistance from Auris team members was permitted to assist bronchoscopists during the procedures.

\section{Procedures}

All cadavers were intubated and mechanically ventilated during the procedures. Each operator performed bronchoscopy on 1 dedicated cadaver using the robotic bronchoscopic platform. When targeting peripheral lesions, the outer sheath and inner scope were advanced to the orifice of the targeted bronchus where the outer sheath was "parked." The inner scope was subsequently advanced further into the lung parenchyma towards targeted lesions using a combination of direct endoscopic visualization, electromagnetic navigational guidance, and fluoroscopy (Fig. 3). Once the bronchoscopist was positioned at the estimated location of the 
Table 1. Lobar distribution of peripheral lesions

\begin{tabular}{lr}
\hline Lobe & $n$ \\
\hline RUL & 14 \\
RML & 5 \\
RLL & 14 \\
LUL & 13 \\
Lingula & 10 \\
LLL & 11 \\
\hline Total & 67 \\
\hline
\end{tabular}

targeted lesion, radial probe endobronchial ultrasound (UM S20$17 \mathrm{~S}$, Olympus, Tokyo, Japan) was introduced through the working channel of the system and was used to survey the lung parenchyma and confirm lesion localization. Radial ultrasound images were recorded as "concentric" when the ultrasound representation of the targeted lesion surrounded the radial EBUS probe and "eccentric" when the ultrasound image was biased to one side of the radial EBUS probe with no component of the lesion surrounding the probe (Fig. 4).

\section{Biopsy}

Following lesion localization, transbronchial needle aspiration (TBNA) was performed in all cases using a 24-G TBNA needle (Auris Health). Specimens were immediately evaluated using light microscopy and were recorded as "diagnostic" when pigmented mica powder was observed within aspirated specimens and "non-diagnostic" when pigmented powder was absent (Fig. 5). Needles were thoroughly rinsed with saline and purged with air between aspirates to limit the possibility of cross-contamination. Following TBNA, transbronchial biopsies were obtained using flexible forceps at the discretion of the bronchoscopist.

\section{Statistical Analysis}

Continuous data are presented as the mean (standard deviation, SD) and the median (range). The Fisher exact test was used to determine the significance of $2 \times 2$ contingency tables. Statistical analyses were performed using JMP 14 software.

\section{Results}

\section{Cadaver and Nodule Characteristics}

Four male and 4 female fresh cadavers were prepared with a mean height of $170 \mathrm{~cm}(\mathrm{SD} 11.1 \mathrm{~cm}$, range 152188). Sixty-seven nodules were evaluated in the 8 prepared cadavers. The mean nodule size, measured as the largest axial diameter by CT scan, was $20.4 \mathrm{~mm}$ (range 9.6-28.3), with 45/67 (68\%) of the nodules measuring 10$20 \mathrm{~mm}$ in diameter and 21/67 (31\%) of the nodules measuring $21-30 \mathrm{~mm}$.

Robotic Bronchoscopy for Simulated Tumor Targets
Table 2. Diagnostic yield characteristics

\begin{tabular}{llc}
\hline Characteristic & Diagnostic yield, $n / N(\%)$ & $p$ value \\
\hline Size & & \\
$\quad$ Overall & $65 / 67(97)$ & \\
$\quad<20 \mathrm{~mm}$ & $44 / 46(95.7)$ & $>0.999$ \\
$\quad>21 \mathrm{~mm}$ & $21 / 21(100)$ & \\
Ultrasound & & \\
$\quad$ Concentric & $10 / 11(90.9)$ & 0.341 \\
$\quad$ Eccentric & $47 / 48(97.9)$ & \\
Distance from pleura & & \\
$\quad<10 \mathrm{~mm}$ & $18 / 20(90)$ & 0.086 \\
$\quad>11 \mathrm{~mm}$ & $47 / 47(100)$ & \\
\hline
\end{tabular}

The mean and median distance from the closest edge of targeted nodules to the nearest pleural edge was 16.0 $\mathrm{mm}$ (SD 10.6) and $13.8 \mathrm{~mm}$ (range $0.0-35.3$ ), respectively; 36/67 (53.7\%) of the nodules were visible under fluoroscopy. On average, the robotic scope was advanced to within $27.6 \mathrm{~mm}$ (SD 8.6) of targeted lesions. Fifty-nine of $67(88.1 \%)$ nodules were visualized using radial probe endobronchial ultrasound, with 48/59 (81.4\%) nodules demonstrating eccentric ultrasound images and 11/59 (18.6\%) demonstrating concentric ultrasound images. Table 1 lists the lobar distribution of all nodules.

\section{Diagnostic Yield}

The diagnostic yield of robotic-assisted bronchoscopic TBNA for all nodules was 63/67 (94\%). Transbronchial forceps biopsy was performed in 11 nodules and provided the diagnosis for 2 cases in which TBNA was non-diagnostic, producing an overall diagnostic yield of $65 / 67$ (97\%). For nodules measuring 21-30 mm, the diagnostic yield was $21 / 21$ (100\%); for nodules smaller than $20 \mathrm{~mm}$, the diagnostic yield was $44 / 46$ (95.7\%; $p$ > $0.999)$. For lesions with an eccentric ultrasound view, the diagnostic yield was $47 / 48$ (97.9\%) compared with 10/11 $(90.9 \%)$ in patients with a concentric view $(p=0.341)$. The diagnostic yield for nodules $<10 \mathrm{~mm}$ from the pleura was $18 / 20$ (90\%) compared with $47 / 47$ (100\%) nodules $\geq 10 \mathrm{~mm}$ from the nearest pleural surface $(p=0.086$; Table 2).

\section{Airway Trauma}

Cadaveric airways were thoroughly inspected during navigation to targeted lesions as well as following biopsies during removal of the robotic scope from the distal bronchus. No obvious airway trauma was identified during study procedures. 


\section{Discussion}

An estimated 1.6 million new pulmonary nodules will be detected by chest CT scans annually in the USA. While most of these findings will be followed with surveillance imaging, many will require a tissue diagnosis [10]. The diagnostic yield of current bronchoscopic approaches for pulmonary nodules $10-30 \mathrm{~mm}$ in size continues to challenge clinicians, and with the broadened adoption of lung cancer screening there may be increasing demands placed on bronchoscopists to offer diagnostic procedures using safe and minimally invasive techniques. Despite increased experience using approaches such as electromagnetic navigation and radial probe endobronchial ultrasound, the diagnostic yield in this patient population may be suboptimal. A recent prospective, randomized controlled trial comparing conventional bronchoscopy with fluoroscopic guidance to bronchoscopy using a thin scope and radial probe endobronchial ultrasound failed to show a statistically significant difference between groups in patients with peripheral pulmonary lesions $1.5-5 \mathrm{~cm}$ in size, with an overall diagnostic yield of less than $50 \%$ [11].

Some potential limitations of guided techniques using conventional bronchoscopes may be reach into the lung periphery and control and precision at the time of biopsy. Another limiting factor may be the anatomical relationships of peripheral lesions and the segmental bronchi that are used to access them. Several studies using radial probe endobronchial ultrasound and conventional bronchoscopy have demonstrated diagnostic yields of $80 \%$ or greater with a concentric ultrasound image which is obtained when the nodule surrounds the bronchus, compared with yields of $30-40 \%$ with an eccentric ultrasound image that indicates that the nodule is adjacent to the bronchus [12]. A review of nearly 300 patients demonstrated eccentric ultrasound views in nearly half of patients, suggesting that improved control and dexterity of instruments at the time of biopsy may improve the diagnostic yield in a significant proportion of patients with peripheral lesions [13].

Recently, robotic endoscopic systems have been introduced as platforms to potentially improve diagnostic yields for peripheral lesion biopsy. These systems utilize uniquely designed catheters and scopes that incorporate multiple articulation points which may influence procedural characteristics during bronchoscopy. The outer sheath of the robotic platform may provide additional structural support that facilitates advancement of the inner scope further into the lung parenchyma, and the ability to maneuver and control the distal end of the inner scope through multiple active articulation points may provide the bronchoscopist with the ability to aim biopsy instruments with greater precision than with conventional approaches. Additionally, these platforms incorporate image guidance such as electromagnetic navigation and have working channels compatible with radial ultrasound probes and conventional biopsy tools.

Despite these potential advantages, robotic-assisted bronchoscopy is in its early phases and relatively little has been published regarding how these systems may affect procedural outcomes. We have previously reported that a robotic endoscopic system comprised of a $6.0-\mathrm{mm}$ outer sheath and scope with an outer diameter of $4.4 \mathrm{~mm}$ was able to reach further into the lung periphery than similarly sized conventional thin bronchoscopes. This current study was performed to evaluate the potential clinical implications of added reach, along with control and maneuverability for biopsying peripheral nodules $10-30 \mathrm{~mm}$ in diameter in cadaveric lungs.

In this study, the robotic endoscopic system was successful at biopsying peripheral lesions in the cadaveric model regardless of size (between 10 and $30 \mathrm{~mm}$ ), location relative to the pleura, or the presence of an eccentric or concentric radial ultrasound view. This last metric may be of particular clinical significance as the ability to successfully biopsy lesions which are adjacent to peripheral bronchi is suboptimal at this time using contemporary bronchoscopic techniques. The success of peripheral lesion biopsy in this study may be due to factors such as reach into the lung periphery, as well as scope control at the time of biopsy. For lesions that were beyond $20 \mathrm{~mm}$ of the tip of the robotic system, the ability to articulate along multiple points of the scope in concert with navigational alignment may assist with passing biopsy instruments directly into targeted lesions.

Potential limitations to interpreting the applicability of these findings should begin with assessment of the models themselves. Despite the inclusion of mechanical ventilation, fluoroscopy, electromagnetic navigation, radial ultrasound, and cadavers with intact head, neck, shoulders, and torsos to simulate live human procedures, cadaver tissue may respond differently than live human tissue. The preserved and cooled nature of cadavers may not be reflective of dynamic changes associated with bronchoscope manipulation and respiratory variation in live subjects. While no overt airway trauma was observed during the bronchoscopy procedures, the effect of robotic bronchoscopes on live human airway tissue will best be defined through ongoing human pilot studies utilizing the system. Additionally, targeted lesions in this study 
were artificially created, and therefore differ from naturally occurring pulmonary nodules in live human subjects. Despite this, all efforts were made to produce peripheral lesions that closely mimicked pulmonary nodules. Lesion size was carefully controlled by limiting the volume of injected material and anecdotal feedback from all bronchoscopists was that these lesions very closely simulated the appearance of peripheral pulmonary nodules in patients. To minimize potential bias and confounding factors, the bronchoscopists were not involved in any portion of cadaver preparation, including implantation of the tumor targets.

As a feasibility study of new technology, the specific aim was to evaluate the ability of the robotic system to locate and biopsy peripheral lesions in human lungs in a controlled environment. The inability to reach, localize, and biopsy peripheral lesions in a controlled setting using the robotic system would likely preclude the ability to further investigate the platform in live human subjects. As such, the focus of this study was on feasibility of the robotic system alone and the omission of a control arm using conventional bronchoscopic biopsy was intentional; any comparison to current bronchoscopic practices will be better determined in prospective comparative trials with live human subjects.

In conclusion, the robotic bronchoscopic system used in this study was successful at biopsying $97 \%$ of peripheral pulmonary lesions $10-30 \mathrm{~mm}$ in size in human ca- davers. These results support the idea that this platform may potentially improve the diagnostic yield of peripheral pulmonary lesions and merits the progression to studies in live human subjects to further explore these findings.

\section{Statement of Ethics}

The authors have no ethical conflicts to disclose.

\section{Disclosure Statement}

Alexander Chen, Nicholas Pastis, Michael Machuzak, Thomas Gildea, Michael Simoff, Colin Gillespie, Amit Mahajan, and Scott Oh have received consulting fees from Auris Health. Gerard Silvestri has received research support from Auris Health.

\section{Author Contributions}

A.C.C., N.J.P., M.S.M., T.R.G., M.J.S., C.T.G., A.K.M., S.S.O., and G.A.S. each made substantial contributions to the conception or design of the work, the acquisition, analysis, or interpretation of data for the work, drafted and assisted in critical revisions to the work for important intellectual content, provided final approval of the version to be published, and are in agreement to be accountable for all aspects of the work in ensuring that questions related to the accuracy or integrity of any part of the work are appropriately investigated and resolved.

\section{References}

1 Ost DE, Ernst A, Lei X, Kovitz KL, Benzaquen S, Diaz-Mendoza J, et al.; AQuIRE Bronchoscopy Registry. Diagnostic yield and complications of bronchoscopy for peripheral lung lesions. Results of the AQuIRE Registry. Am J Respir Crit Care Med. 2016 Jan;193(1):68-77.

2 Silvestri GA, Vachani A, Whitney D, Elashoff M, Porta Smith K, Ferguson JS, et al.; AEGIS Study Team. A bronchial genomic classifier for the diagnostic evaluation of lung cancer. N Engl J Med. 2015 Jul;373(3):243-51.

3 Kurimoto N, Miyazawa T, Okimasa S, Maeda A, Oiwa H, Miyazu Y, et al. Endobronchial ultrasonography using a guide sheath increases the ability to diagnose peripheral pulmonary lesions endoscopically. Chest. 2004 Sep; 126(3):959-65.

4 Yamada N, Yamazaki K, Kurimoto N, Asahina $\mathrm{H}$, Kikuchi E, Shinagawa N, et al. Factors related to diagnostic yield of transbronchial biopsy using endobronchial ultrasonography with a guide sheath in small peripheral pulmonary lesions. Chest. 2007 Aug;132(2):6038.

Robotic Bronchoscopy for Simulated Tumor Targets
5 Chen A, Chenna P, Loiselle A, Massoni J, Mayse M, Misselhorn D. Radial probe endobronchial ultrasound for peripheral pulmonary lesions. A 5-year institutional experience. Ann Am Thorac Soc. 2014 May;11(4): $578-82$.

6 Rojas-Solano JR, Ugalde-Gamboa L, Machuzak M. Robotic bronchoscopy for diagnosis of suspected lung cancer: A feasibility study. J Bronchology Interv Pulmonol. 2018 Jul; 25(3):168-75

7 Fielding DI, Bashirzadeh F, Son JH, Todman M, Chin A, Tan L, et al. First human use of a new robotic-assisted fiber optic sensing navigation system for small peripheral pulmonary nodules. Respiration. 2019;98(2):142-50.

8 Chen AC, Gillespie CT. Robotic endoscopic airway challenge: REACH assessment. Ann Thorac Surg. 2018 Jul;106(1):293-7.

9 Chen A, Machuzak M, Edell E, Silvestri GA. Peripheral bronchoscopy training using a human cadaveric model and simulated tumor targets. J Bronchology Interv Pulmonol. 2016 Jan;23(1):83-6.
10 Gould MK, Tang T, Liu IL, Lee J, Zheng C, Danforth KN, et al. Recent trends in the identification of incidental pulmonary nodules. Am J Respir Crit Care Med. 2015 Nov; 192(10):1208-14.

11 Tanner NT, Yarmus L, Chen A, Wang Memoli J, Mehta HJ, Pastis NJ, et al. Standard bronchoscopy with fluoroscopy vs thin bronchoscopy and radial endobronchial ultrasound for biopsy of pulmonary lesions: A multicenter, prospective, randomized trial. Chest. 2018 Nov; 154(5):1035-43.

12 Ali MS, Trick W, Mba BI, Mohananey D, Sethi J, Musani AI. Radial endobronchial ultrasound for the diagnosis of peripheral pulmonary lesions: A systematic review and meta-analysis. Respirology. 2017 Apr;22(3):44353.

13 Chen AC, Loiselle A, Zhou L, Baty J, Misselhorn D. Localization of peripheral pulmonary lesions using a method of computed tomography-anatomic correlation and radial probe endobronchial ultrasound confirmation. Ann Am Thorac Soc. 2016;13(9):1586-92. 